\title{
Generalized Ulam-Hyers-Rassias Stability Results of Solution for Nonlinear Fractional Differential Problem with Boundary Conditions
}

\author{
A. Naimi, ${ }^{1}$ B. Tellab, ${ }^{1}$ Y. Altayeb ${ }^{1},{ }^{2}$ and A. Moumen ${ }^{3}$ \\ ${ }^{1}$ Laboratory of Applied Mathematics, Kasdi Merbah University, B. P. 511, 30000 Ouargla, Algeria \\ ${ }^{2}$ Department of Mathematics, College of Sciences and Arts, Qassim University, Ar-Rass, Saudi Arabia \\ ${ }^{3}$ Department of Mathematics, Faculty of Science, University of Hail, Hail 55425, Saudi Arabia
}

Correspondence should be addressed to Y. Altayeb; y.hassan@qu.edu.sa

Received 9 September 2021; Accepted 3 November 2021; Published 23 November 2021

Academic Editor: Adel Ouannas

Copyright (c) 2021 A. Naimi et al. This is an open access article distributed under the Creative Commons Attribution License, which permits unrestricted use, distribution, and reproduction in any medium, provided the original work is properly cited.

The problem of existence and generalized Ulam-Hyers-Rassias stability results for fractional differential equation with boundary conditions on unbounded interval is considered. Based on Schauder's fixed point theorem, the existence and generalized Ulam-Hyers-Rassias stability results are proved, and then some examples are given to illustrate our main results.

\section{Introduction and Position of Problem}

There are various, not equivalent, definitions of fractional derivatives according to Grunwald Letnikov, Weil, Caputo, and Riemann-Liouville, etc. Ordinary and partial differential order equations (with fractional derivatives of Caputo and Riemann-Liouville) have awakened in recent years with considerable interest both in mathematics and in applications. Let us describe the abstract Cauchy problem:

$$
\begin{aligned}
D_{t}^{\gamma} u(t) & =A u(t), \quad m-1<\gamma \leq m \in \mathbb{N}, \\
\frac{\mathrm{d}^{\kappa}}{\mathrm{d} t^{\kappa}} u(0) & =\xi_{\kappa}, \quad \kappa=0, \ldots, m-1,
\end{aligned}
$$

where the corresponding solutions are represented through the Mittag-Leffler function. In mathematical papers on fractional differential equations, the Riemann-Liouville approach to the concept of a fractional order derivative $\gamma \geq 0$ is usually used as follows:

$$
\begin{array}{r}
D^{\gamma} u(t)=\left(\frac{\mathrm{d}}{\mathrm{d} t}\right)^{m} \frac{1}{\Gamma(m-\gamma)} \int_{0}^{t}(t-r)^{m-\gamma-1} u(r) \mathrm{d} r, \\
m-1<\gamma \leq m \in \mathbb{N} .
\end{array}
$$

The fractional Riemann-Liouville derivative is the left inverse to the corresponding fractional integral, which is a natural generalization of the Cauchy formula for the antiderivative function $u(t)$. The initial conditions, of the initial value problem for ordinary differential equations of fractional order $\gamma$ with fractional derivatives in the Riemann-Liouville form, are given in terms of fractional integrals:

$$
\begin{array}{r}
\frac{1}{\Gamma(m-\kappa-\gamma)} \int_{0}^{0_{+}}\left(0_{+}-r\right)^{m-\kappa-\gamma-1} u^{(\kappa)}(r) \mathrm{d} r, \\
\kappa=0, \ldots, m-1 .
\end{array}
$$

To satisfy the physical requirements, Caputo introduced an alternative definition of the fractional differential derivative. It was adopted by Caputo and Mainardi as 


$$
\begin{array}{r}
{ }^{c} D_{t}^{\gamma} u(t)=\frac{1}{\Gamma(m-\gamma)} \int_{0}^{t}(t-r)^{m-\gamma-1} u^{(m)}(r) \mathrm{d} r, \\
m-1<\gamma \leq m .
\end{array}
$$

The advantage of this definition is a more natural solution for the problem of initial conditions for solving integro-differential equations of noninteger orders.

The cases of Caputo derivative for $0<\gamma<1$ was called the regularized fractional derivative of order $\gamma$.

This paper concerns the existence with Ulam stability for the following equation:

$$
D_{0^{+}}^{\beta} u(t)+f(t, u(t))+\theta(t) g(u(t))=0, \quad t \geq 0 .
$$

For a continuous function $u(t)$ together with boundary conditions,

$$
\begin{aligned}
u(0) & =0 \\
u^{\prime}(0) & =0 \\
D_{0^{+}}^{\beta-1} u(+\infty) & =b u(\xi)+\lambda \int_{0}^{\sigma} u(s) \mathrm{d} s,
\end{aligned}
$$

where $2<\beta \leq 3,0 \leq \lambda, b<\infty$, we fix $0 \leq \xi<\sigma<\infty$, the functions $f: \mathbb{R}_{+} \times \mathbb{R} \longrightarrow \mathbb{R}$ and $g: \mathbb{R} \longrightarrow \mathbb{R}$ are continuous and $\theta$ is a continuous decreasing positive function such that $0<\theta(t) \leq 1$, for all $t \in[0,+\infty) . D_{0^{+}}^{\beta}$ is the standard Riemann-Liouville fractional derivative of order $\beta$.

\section{Literature Overview}

Fractional differential equations, which are often encountered in mathematical modeling of various processes in natural and technical sciences, play an important role in describing many phenomena in physics, bioengineering, and engineering applications. The properties of such equations were investigated in many reviews (among them, we refer [1-6]).

Regarding the existence, we mention the work by Zhao and Ge [7], where the authors used the well-known Leray Schauder nonlinear alternative theorem to prove the existence of positive solutions to the problem

$$
\begin{cases}D_{0^{+}}^{\alpha} u(t)+f(t, u(t))=0, & t \in[0,+\infty), 1<\alpha \leq 2, \\ u(0)=0, & D_{0^{+}}^{\alpha-1} u(+\infty)=\beta u(\xi)\end{cases}
$$

where $f \in C([0,+\infty) \times \mathbb{R},[0,+\infty)), 0 \leq \xi, \beta<+\infty$. Next, Wang et al. [8] extended the above results and discussed the question of existence for solutions of (7) with condition:

$$
D_{0^{+}}^{\alpha-1} u(+\infty)=\lambda \int_{0}^{\tau} u(s) \mathrm{d} s
$$

where $0 \leq \lambda, \tau<+\infty$. Shen et al. [9] considered the existence of solution for boundary value problem of nonlinear multipoint fractional differential equation:

$$
\begin{cases}D_{0^{+}}^{\gamma} u(t)=f\left(t, u(t), D_{0^{+}}^{\gamma} u(t)\right), & t \in[0,+\infty), \\ u(0)=0, u^{\prime}(0)=0, & D_{0^{+}}^{\gamma-1} u(+\infty)=\sum_{i=1}^{m-2} \beta_{i} u\left(\xi_{i}\right)\end{cases}
$$

where

$$
\begin{gathered}
2<\gamma \leq 3, \\
f \in C\left(\mathbb{R}_{+} \times \mathbb{R} \times \mathbb{R}, \mathbb{R}\right), \\
\Gamma(\beta)-\sum_{i=1}^{m-2} \beta_{i} \xi_{i}^{\gamma-1} .
\end{gathered}
$$

SM Ulam in 1940 was the first to raise the question of stability for functional equations. After his lecture, this question became popular for many specialists in mathematical analysis. It became an area of in-depth research (see for more details [10-12]). Next, many mathematicians turned in their studies to two types of stability-according to Ulam-Hyers and according to Ulam-Hyers-Rassias. This kind of study has become one of the central and most important in the fields of fractional differential equations. Details of recent advances in Ulam-Hyers sustainability and according to Ulam-Hyers for differential equations can be found in [13, 14] and in articles [15-17]. However, as far as we know, most authors discussed Ulam stability of some fractional differential problem on bounded/unbounded intervals, while the present paper discusses the existence of solutions and stability in the sense of Ulam-Hyers-Rassias for nonlinear fractional differential equations boundary conditions, for which research is just beginning, please see [18-23].

\section{Preliminaries}

Here, we present some notations, definitions, auxiliary lemmas concerning fractional calculus, fixed point theorems, and some preliminary concepts of fractional calculus.

Definition 1 (see $[4,24]$ ). The Riemann-Liouville fractional integral of order $\beta$ for a function $f$ is defined as

$$
I_{0^{+}}^{\beta} f(t)=\frac{1}{\Gamma(\beta)} \int_{0}^{t}(t-s)^{\beta-1} f(s) \mathrm{d} s,
$$

provided the right side is pointwise defined on $(0 ;+\infty)$.

Lemma 1 (see [25]). Let $U \subset X$ be a bounded set. $U$ is said to be relatively compact in a space $E$ if

(i) $\forall u \in U$, the function $u(t) /\left(1+t^{\beta-1}\right)$ is equicontinuous on any compact subinterval of $J$.

(ii) $\forall \varepsilon>0$, there exists a constant $T=T(\varepsilon)>0$ such that

$$
\left|\frac{u\left(t_{2}\right)}{1+t_{2}^{\beta-1}}-\frac{u\left(t_{1}\right)}{1+t_{1}^{\beta-1}}\right|<\varepsilon, \quad t_{1}, t_{2} \geq T \text { and } u \in U
$$


Lemma 2 (see $[4,24]$ ). Assume that $x \in C(J) \cap L^{1}(J)$ with a fractional derivative of order $\beta>0$. Then,

$$
I_{0^{+}}^{\beta} D_{0^{+}}^{\beta} x(t)=x(t)-C_{1} t^{\beta-1}-C_{2} t^{\beta-2}-\cdots-C_{n} t^{\beta-n},
$$

where $C_{1}, C_{2}, \ldots, C_{N} \in \mathbb{R}$ with $n=[\beta]+1$.

Lemma 3. Let us define the following space:

$$
E=\left\{u \in C[0,+\infty): \sup _{t \geq 0} \frac{|u(t)|}{1+t^{\beta-1}}<+\infty\right\},
$$

equipped with the norm

$$
\|u\|_{E}=\sup _{t \geq 0} \frac{|u(t)|}{1+t^{\beta-1}} .
$$

Then, clearly $\left(E,\|\cdot\|_{E}\right)$ is a Banach space.

Lemma 4. $u$ is a solution of the problem (5)-(6) if and only if $u$ satisfies the following integral equation:

$$
u(t)=\int_{0}^{+\infty} H(t, s)[f(s, u(s))+\theta(s) g(u(s))] \mathrm{d} s,
$$

where

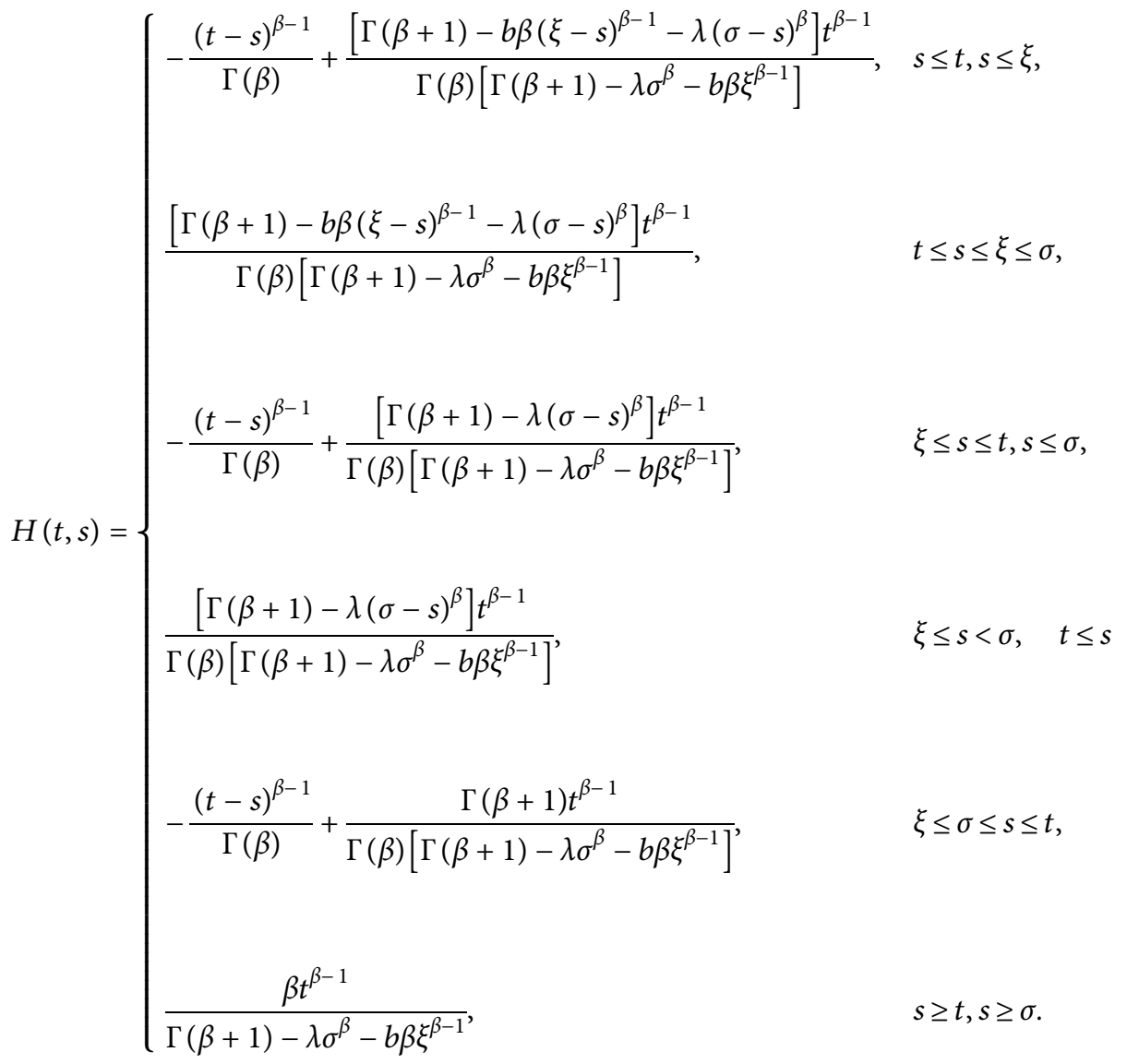

Proof. Using Lemma 2, we have

$$
\begin{aligned}
u(t)= & -I_{0^{+}}^{\beta}[f(t, u(t))+\theta(t) g(u(t))] \\
& +c_{1} t^{\beta-1}+c_{2} t^{\beta-2}+c_{3} t^{\beta-3} .
\end{aligned}
$$

By the first and second conditions, we get

$$
\begin{aligned}
& c_{3}=0, \\
& c_{2}=0 .
\end{aligned}
$$

$$
u(t)=-I_{0^{+}}^{\beta}[f(t, u(t))+\theta(t) g(u(t))]+c_{1} t^{\beta-1} .
$$

From the third boundary condition, it follows that

$$
\begin{aligned}
D_{0^{+}}^{\beta-1} u(t) & =-I_{0^{+}}^{\beta-\beta+1}[f(t, u(t))+\theta(t) g(u(t))]+c_{1} \Gamma(\beta) \\
& =-\int_{0}^{t}[f(s, u(s))+\theta(s) g(u(s))] \mathrm{d} s+c_{1} \Gamma(\beta) .
\end{aligned}
$$

Consequently,
On the other hand, we have 


$$
\begin{aligned}
b u(\xi)+\lambda \int_{0}^{\sigma} u(s) \mathrm{d} s= & -b I_{0^{+}}^{\beta}[f(\xi, u(\xi))+\theta(\xi) g(u(\xi))]+c_{1} b \xi^{\beta-1} \\
& -\lambda \int_{0}^{\sigma} I_{0^{+}}^{\beta}[f(s, u(s))+\theta(s) g(u(s))] \mathrm{d} s+\lambda \int_{0}^{\sigma} c_{1} s^{\beta-1} \mathrm{~d} s \\
= & -b I_{0^{+}}^{\beta}[f(\xi, u(\xi))+\theta(\xi) g(u(\xi))]+c_{1} b \xi^{\beta-1} \\
& -\lambda I_{0^{+}}^{\beta+1}[f(\sigma, u(\sigma))+\theta(\sigma) g(u(\sigma))]+\frac{\lambda \sigma^{\beta}}{\beta} c_{1} .
\end{aligned}
$$

Then, we deduce

$$
\begin{aligned}
c_{1}= & \frac{\beta}{\Gamma(\beta+1)-\lambda \sigma^{\beta}-b \beta \xi^{\beta-1}}\left[\int_{0}^{+\infty}[f(s, u(s))+\theta(s) g(u(s))] \mathrm{d} s\right. \\
& \left.-b I_{0^{+}}^{\beta}[f(\xi, u(\xi))+g(u(\xi))]-\lambda I_{0^{+}}^{\beta+1}[f(\sigma, u(\sigma))+\theta(\sigma) g(u(\sigma))]\right] .
\end{aligned}
$$

By substituting the values of $c_{1}, c_{2}$, and $c_{3}$ in (18), we get the following integral equation:

$$
\begin{aligned}
u(t)= & -\int_{0}^{t} \frac{(t-s)^{\beta-1}}{\Gamma(\beta)}[f(s, u(s))+\theta(s) g(u(s))] \mathrm{d} s \\
& +\frac{\beta t^{\beta-1}}{\Gamma(\beta+1)-\lambda \sigma^{\beta}-b \beta \xi^{\beta-1}} \int_{0}^{+\infty}[f(s, u(s))+\theta(s) g(u(s))] \mathrm{d} s \\
& -\frac{b \beta t^{\beta-1}}{\Gamma(\beta+1)-\lambda \sigma^{\beta}-b \beta \xi^{\beta-1}} \int_{0}^{\xi} \frac{(\xi-s)^{\beta-1}}{\Gamma(\beta)}[f(s, u(s))+\theta(s) g(u(s))] \mathrm{d} s \\
& -\frac{\lambda \beta t^{\beta-1}}{\Gamma(\beta+1)-\lambda \sigma^{\beta}-b \beta \xi^{\beta-1}} \int_{0}^{\sigma} \frac{(\sigma-s)^{\beta}}{\Gamma(\beta+1)}[f(s, u(s))+\theta(s) g(u(s))] \mathrm{d} s .
\end{aligned}
$$

Then, we get (16).

Conversely, suppose that (16) is satisfied. To get (5), we use the following appropriate relationships:

$$
\begin{aligned}
D_{0^{+}}^{\beta} I_{0^{+}}^{\beta}[f(t, u(t))+\theta(t) g(u(t))] & =f(t, u(t))+\theta(t) g(u(t)), \\
D_{0^{+}}^{\beta} t^{\beta-1} & =0 .
\end{aligned}
$$

The present paper is organized as follows. In Section 4, we prove the existence of the solution for problem (5)-(6) in the Banach space. The generalized Ulam-Hyers stable is stated and proved in Section 5. Finally, an illustrative example is given.

\section{Existence Result}

In order to prove the existence of the solution for problem (5)-(6), we transform problem (5)-(6) into the fixed point problem $P u=u$, where $P$ is an operator defined on

$$
\mathfrak{B}(r)=\left\{u \in E, \quad\|u\|_{E} \leq r\right\},
$$

by

$$
P u(t)=\int_{0}^{+\infty} H(t, s)[f(s, u(s))+\theta(s) g(u(s))] \mathrm{d} s .
$$

Theorem 1. Let $f:[0,+\infty) \times \mathbb{R} \longrightarrow \mathbb{R}$ and $g: \mathbb{R} \longrightarrow \mathbb{R}$ are two functions such that 
$\left(A_{1}\right) \Gamma(\beta+1)>\lambda \sigma^{\beta}+b \beta \xi^{\beta-1}$.

$\left(A_{2}\right)$ There exist a nonnegative measurable function $\psi_{1}$ defined on $[0,+\infty)$ and a real constant $L>0$ such that:

$|f(t, u(t))-f(t, v(t))| \leq \psi_{1}(t)|u(t)-v(t)|, \quad u, v \in \mathbb{R}$,

$|g(u(t))-g(v(t))| \leq L|u(t)-v(t)|, \quad u, v \in \mathbb{R}$,

$\beta \int_{0}^{+\infty}\left(1+t^{\beta-1}\right)\left[\psi_{1}(t)+\psi_{2}(t)\right] \mathrm{d} t<\Gamma(\beta+1)-\lambda \sigma^{\beta}-b \beta \xi^{\beta-1}$

with

$$
\psi_{2}(t)=\theta(t) L, \quad \text { for each } t \in[0,+\infty) .
$$

$\left(A_{3}\right)$ Let $\phi_{1}(t)=|f(t, 0)|$ and $\phi_{2}(t)=\theta(t)|g(0)|$, $t \in[0,+\infty)$ such that

$$
\int_{0}^{+\infty}\left[\phi_{1}(t)+\phi_{2}(t)\right] \mathrm{d} t<+\infty
$$

Then, problem (5)-(6) has at least one solution in $[0,+\infty)$.

Lemma 5. If $\left(A_{1}\right)$ holds, then the Green function $H(t, s)$ satisfiesfor all $\xi, \sigma, s, t \in[0,+\infty)$, we have

$$
\frac{H(t, s)}{1+t^{\beta-1}} \leq \frac{\beta}{\Gamma(\beta+1)-\lambda \sigma^{\beta}-b \beta \xi^{\beta-1}} .
$$

Proof. If $s \leq t$, and $s \leq \xi$, we get

$$
\begin{aligned}
\frac{H(t, s)}{1+t^{\beta-1}}= & -\frac{(t-s)^{\beta-1}}{\left(1+t^{\beta-1}\right) \Gamma(\beta)} \\
& +\frac{\left[\Gamma(\beta+1)-b \beta(\xi-s)^{\beta-1}-\lambda(\sigma-s)^{\beta}\right] t^{\beta-1}}{\Gamma(\beta)\left[\Gamma(\beta+1)-\lambda \sigma^{\beta}-b \beta \xi^{\beta-1}\right]\left(1+t^{\beta-1}\right)} \\
& \leq \frac{\Gamma(\beta+1) t^{\beta-1}}{\Gamma(\beta)\left[\Gamma(\beta+1)-\lambda \sigma^{\beta}-b \beta \xi^{\beta-1}\right]\left(1+t^{\beta-1}\right)} \\
& \leq \frac{\beta}{\Gamma(\beta+1)-\lambda \sigma^{\beta}-b \beta \xi^{\beta-1}} .
\end{aligned}
$$

All other cases of $H(t, s)$ are simple. This completes the proof of Lemma 5.

Proof. of Theorem 1. We shall use Schauder's fixed point theorem, which is divided into three steps.

Step 1. Let $r>0$ such that

$$
r \geq \frac{\beta \int_{0}^{+\infty}\left[\phi_{1}(p)+\phi_{2}(p)\right] \mathrm{d} p}{\Gamma(\beta+1)-\lambda \sigma^{\beta}-b \beta \xi^{\beta-1}-\beta \int_{0}^{+\infty}\left(1+p^{\beta-1}\right)\left[\psi_{1}(p)+\psi_{2}(p)\right] \mathrm{d} p} .
$$

If $u$ is a continuous function on $J$, then $P u \in C(J)$. In order to show $P\left(\mathfrak{B}_{r}\right) \subset \mathfrak{B}_{r}$, let $u \in \mathfrak{B}_{r}, t \in \mathbb{R}^{+}$. Then,

$$
\begin{aligned}
& \left|\frac{P u(t)}{1+t^{\beta-1}}\right|=\left|\int_{0}^{+\infty} \frac{H(t, s)}{1+t^{\beta-1}}[f(s, u(s))+\theta(s) g(u(s))] \mathrm{d} s\right| \\
& \leq \frac{\beta}{\Gamma(\beta+1)-\lambda \sigma^{\beta}-b \beta \xi^{\beta-1}}\left|\int_{0}^{+\infty}[f(s, u(s))+\theta(s) g(u(s))] \mathrm{d} s\right| \\
& \leq \frac{\beta}{\Gamma(\beta+1)-\lambda \sigma^{\beta}-b \beta \xi^{\beta-1}} \times \\
& \cdot \int_{0}^{+\infty}[|f(s, u(s))-f(s, 0)|+\theta(s)|g(u(s))-g(0)|+|f(s, 0)|+|\theta(s) g(0)|] \mathrm{d} s \\
& \leq \frac{\beta}{\Gamma(\beta+1)-\lambda \sigma^{\beta}-b \beta \xi^{\beta-1}} \int_{0}^{+\infty}\left[\psi_{1}(s)|u(s)|+\theta(s) L|u(s)|+\phi_{1}(s)+\phi_{2}(s)\right] \mathrm{d} s \\
& \leq \frac{\beta}{\Gamma(\beta+1)-\lambda \sigma^{\beta}-b \beta \xi^{\beta-1}} \int_{0}^{+\infty}\left[\phi_{1}(s)+\phi_{2}(s)\right] \mathrm{d} s \\
& +\frac{\beta\|u\|_{E}}{\Gamma(\beta+1)-\lambda \sigma^{\beta}-b \beta \xi^{\beta-1}} \int_{0}^{+\infty}\left(1+s^{\beta-1}\right)\left[\psi_{1}(s)+\psi_{2}(s)\right] \mathrm{d} s \\
& \leq \frac{\beta}{\Gamma(\beta+1)-\lambda \sigma^{\beta}-b \beta \xi^{\beta-1}} \int_{0}^{+\infty}\left[\phi_{1}(s)+\phi_{2}(s)\right] \mathrm{d} s \\
& +\frac{\beta r}{\Gamma(\beta+1)-\lambda \sigma^{\beta}-b \beta \xi^{\beta-1}} \int_{0}^{+\infty}\left(1+s^{\beta-1}\right)\left[\psi_{1}(s)+\psi_{2}(s)\right] \mathrm{d} s \\
& \leq r \text {. }
\end{aligned}
$$


Therefore, $\|P\|_{E} \leq r$; thus, $P\left(\mathfrak{B}_{r}\right) \in \mathfrak{B}_{r}$.

Step 2. $P: \mathfrak{B}_{r} \longrightarrow \mathfrak{B}_{r}$ is continuous. Let $\left\{u_{n}\right\}$ be a sequence which converges to $u$ in $\mathfrak{B}_{r}$. Then, for all $t \in[0,+\infty)$,

$$
\begin{aligned}
& \left|\frac{P u_{n}(t)-P u(t)}{1+t^{\beta-1}}\right| \\
& =\left|\int_{0}^{+\infty} \frac{H(t, s)}{1+t^{\beta-1}}\left[\left(f\left(s, u_{n}(s)\right)-f(s, u(s))\right)+t \theta n(s) q\left(g\left(u_{n}(s)\right)-g(u(s))\right)\right] \mathrm{d} s\right| \\
& \quad \leq \frac{\beta}{\Gamma(\beta+1)-\lambda \sigma^{\beta}-b \beta \xi^{\beta-1}} \int_{0}^{+\infty}\left|\left(f\left(s, u_{n}(s)\right)-f(s, u(s))\right)+t \theta n(s) q\left(g\left(u_{n}(s)\right)-g(u(s))\right)\right| \mathrm{d} s \\
& \quad \leq \frac{\beta}{\Gamma(\beta+1)-\lambda \sigma^{\beta}-b \beta \xi^{\beta-1}} \int_{0}^{+\infty}\left[f\left(s, u_{n}(s)\right)-f(s, u(s))+\theta g\left(u_{n}(s)\right)-g(u(s))\right] \mathrm{d} s \\
& \quad \leq \frac{\beta\left\|u_{n}-u\right\|_{E}}{\Gamma(\beta+1)-\lambda \sigma^{\beta}-b \beta \xi^{\beta-1}} \int_{0}^{+\infty}\left[\psi_{1}(s)+\psi_{2}(s)\right]\left(1+s^{\beta-1}\right) \mathrm{d} s \\
& \quad<\left\|u_{n}-u\right\|_{E}
\end{aligned}
$$

So, we conclude that $\left\|P u_{n}-P u\right\|_{E} \longrightarrow 0$ as $n \longrightarrow+\infty$. Hence, $P$ is a continuous operator on $E$.

$$
\begin{aligned}
& \left|\frac{P u\left(t_{2}\right)}{1+t_{2}^{\beta-1}}-\frac{P u\left(t_{1}\right)}{1+t_{1}^{\beta-1}}\right| \\
& \quad \leq \int_{0}^{+\infty}\left|\frac{H\left(t_{2}, s\right)}{1+t_{2}^{\beta-1}}-\frac{H\left(t_{1}, s\right)}{1+t_{1}^{\beta-1}}\right||f(s, u(s))+\theta(s) g(u(s))| \mathrm{d} s \\
& \quad \leq \int_{0}^{+\infty}\left|\frac{H\left(t_{2}, s\right)}{1+t_{2}^{\beta-1}}-\frac{H\left(t_{1}, s\right)}{1+t_{1}^{\beta-1}}\right|\left[\left[\psi_{1}(s)+\psi_{2}(s)\right]\left(1+s^{\beta-1}\right)\|u\|_{E}+\phi_{1}(s)+\phi_{2}(s)\right] \mathrm{d} s .
\end{aligned}
$$

Since it is continuous on $J \times J$, we have that $H(t, s) /(1+$ $\left.t^{\beta-1}\right)$ is a uniformly continuous function on the compact set $I \times I$.

For $s \geq t$, the function depends only on $t$, then it is uniformly continuous on $I \times(J / I)$. Therefore, we have $\forall s \in J$ and $t_{1}, t_{2} \in I$; the next property holds.

$\forall \varepsilon>0$, there is $\delta(\varepsilon)>0$ such that, if $\left|t_{1}-t_{2}\right|<\delta$, then

$$
\left|\frac{H\left(t_{2}, s\right)}{1+t_{2}^{\beta-1}}-\frac{H\left(t_{1}, s\right)}{1+t_{1}^{\beta-1}}\right| \leq \varepsilon \text {. }
$$

Step 3. We have two claims to verify that $P\left(\mathfrak{B}_{r}\right)$ is a relatively compact set.

First claim: let $I \subset J$ be a compact interval, $t_{1}, t_{2} \in I$ with $t_{1}<t_{2}$. Then, for any $u \in \mathfrak{B}_{r}$, we have
This property, together with (36) and the fact that

$$
\int_{0}^{+\infty}\left[\left(1+s^{\beta-1}\right)\left[\psi_{1}(s)+\psi_{2}(s)\right] r+\phi_{1}(s)+\phi_{2}(s)\right] \mathrm{d} s<\infty,
$$

means that $P u(t) /\left(1+t^{\beta-1}\right)$ is equicontinuous on $I$.

Second claim: in order to achieve (ii) of Lemma 1, we use 


$$
\begin{aligned}
\lim _{t \rightarrow+\infty} \frac{H(t, s)}{1+t^{\beta-1}}= & \frac{1}{\Gamma(\beta)\left[\Gamma(\beta+1)-\lambda \sigma^{\beta}-b \beta \xi^{\beta-1}\right]} \\
& \times \begin{cases}\lambda\left[\sigma^{\beta}-(\sigma-s)^{\beta}\right]+b \beta\left[\xi^{\beta-1}-(\xi-s)^{\beta-1}\right], & s \leq t, s \leq \xi, \\
\Gamma(\beta+1)-b \beta(\xi-s)^{\beta-1}-\lambda(\sigma-s)^{\beta}, & t \leq s \leq \xi \leq \sigma, \\
\Gamma(\beta+1)-\lambda(\sigma-s)^{\beta}, & \xi \leq s \leq \sigma, \\
\lambda \sigma^{\beta}+b \beta \xi^{\beta-1}, & \xi \leq t, s<\sigma, \\
\beta \Gamma(\beta), & \xi \leq \sigma \leq s \leq t,\end{cases}
\end{aligned}
$$

From (39), it is not hard to see, $\forall \varepsilon>0$, there exists a dependent constant $T=T(\varepsilon)>0$ such that, for $t_{1}, t_{2} \geq T$ and $s \in J$, we have

$$
\left|\frac{H\left(t_{2}, s\right)}{1+t_{2}^{\beta-1}}-\frac{H\left(t_{1}, s\right)}{1+t_{1}^{\beta-1}}\right| \leq \varepsilon .
$$

Now, from (36) and (38), the same property holds for $P u(t) /\left(1+t^{\beta-1}\right)$, uniformly for $u \in \mathfrak{B}_{r}$. Then, $P\left(\mathfrak{B}_{r}\right)$ is equiconvergent at $\infty$.

Thus, Lemma 1 implies that $P\left(\mathfrak{B}_{r}\right)$ is relatively compact.

Therefore, the operator $P$ has a fixed point on $\mathfrak{B}_{r}$. Then, from Schauder's fixed point theorem, we conclude that problem (5)-(6) has at least one solution.

\section{Stability Result}

Before stating and proving our main stability results, let us consider the following integration formula:

$$
v(t)=\int_{0}^{+\infty} H(t, s)[f(s, v(s))+\theta(s) g(v(s))] \mathrm{d} s,
$$

for continuous function $H, \theta$. Here, we suppose that $v \in C([0,+\infty), E)$ has a fractional derivative of order $\beta$, where $2<\beta \leq 3, f:[0,+\infty) \times E \longrightarrow \mathbb{R}$ and $g: E \longrightarrow \mathbb{R}$ are two continuous functions, and let us define the following nonlinear continuous operator:

$$
\begin{gathered}
\mathscr{F}:[0,+\infty) \times E \longrightarrow \mathbb{R}, \\
\mathscr{F} v(t)=D_{0^{+}}^{\beta} v(t)+f(t, v(t))+\theta(t) g(v(t)),
\end{gathered}
$$

Definition 2 (see $[26,27])$. For any $\epsilon>0$ and for each solution $v$ of $(5)-(6)$, such that

$$
\|\mathscr{F} v\|_{E} \leq \epsilon,
$$

problem (5)-(6) is said to be Ulam-Hyers stable, if we can find a positive real number $C_{f}>0$ and a solution $u \in C([0,+\infty), E)$ of (5)-(6) satisfying the inequality

$$
\|u-v\|_{E} \leq \epsilon C_{f} .
$$

Definition 3 (see $[26,27])$. Let $C_{f} \in C\left(\mathbb{R}^{+}, \mathbb{R}^{+}\right)$with $\xi(0)=0$, for each solution $v$ of (5)-(6), we can find a solution $u \in C([0,+\infty), E)$ of (5)-(6) such that

$$
\|u-v\|_{E} \leq C_{f}(\epsilon) .
$$

Then, problem (5)-(6), is called generalized Ulam-Hyers stable.

Definition 4 (see $[26,27])$. For any $\epsilon>0$ for each solution $v$ of (5)-(6), problem (5)-(6) is called Ulam-Hyers-Rassias stable with respect to $\Phi \in C\left([0,+\infty), \mathbb{R}^{+}\right)$if

$$
\|\mathscr{F} v\|_{E} \leq \epsilon \Phi(t) \quad t \in[0,+\infty),
$$

and there exists a real number $C_{f, \Phi}>0$ and a solution $u \in C([0,+\infty), E)$ of (5)-(6) such that

$$
\|u-v\|_{E} \leq \epsilon C_{f, \Phi} \Phi(t), \quad t \in[0,+\infty) .
$$

Definition 5 (see $[26,27])$. For any $\epsilon>0$ and for each solution $v$ of (5)-(6), problem (5)-(6) is called generalized Ulam-Hyers-Rassias stable with respect to $\Phi \in C\left([0,+\infty), \mathbb{R}^{+}\right)$if

$$
\|\mathscr{F} v\|_{E} \leq \Phi(t) \quad t \in[0,+\infty)
$$

and there exists $C_{f, \Phi}>0$ and a solution $u \in C([0,+\infty), E)$ of (5)-(6) such that

$$
\|u-v\|_{E} \leq C_{f, \Phi} \Phi(t), \quad t \in[0,+\infty) .
$$

Theorem 2. If the assumptions $\left(A_{1}\right)$ and $\left(A_{2}\right)$ hold, then problems (5)-(6) are generalized Ulam-Hyers stable.

Proof. By the equivalence between the operators $(I d-P)$ and $\mathscr{F}$ and the assumptions $\left(A_{1}\right),\left(A_{2}\right)$, we find 


$$
\begin{aligned}
|v(t)-u(t)| \leq \mid & |v(t)-P v(t)|+|P v(t)-u(t)| \\
= & |(I d-P) v(t)|+|P v(t)-P u(t)| \\
= & |\mathscr{F} v(t)|+\mid \int_{0}^{+\infty} H(t, s)[f(s, v(s))-f(s, u(s))] \mathrm{d} s \\
& -\int_{0}^{+\infty} H(t, s) \theta(s)[g(v(s))-g(u(s))] \mathrm{d} s \mid \\
& \leq|\mathscr{F} v(t)|+\left|\int_{0}^{+\infty} H(t, s)[f(s, v(s))-f(s, u(s))] \mathrm{d} s\right| \\
& +\left|\int_{0}^{+\infty} H(t, s) \theta(s)[g(v(s))-g(u(s))] \mathrm{d} s\right|
\end{aligned}
$$

Then,

$$
\begin{aligned}
\|v-u\|_{E} \leq & \|\mathscr{F} v\|_{E}+\frac{\beta\|v-u\|_{E}}{\Gamma(\beta+1)-\lambda \sigma^{\beta}-b \beta \xi^{\beta-1}} \times \\
& \cdot \int_{0}^{+\infty}\left[\psi_{1}(s)+\psi_{2}(s)\right]\left(1+s^{1-\beta}\right) \mathrm{d} s \\
\leq & \epsilon+\frac{\beta\|v-u\|_{E}}{\Gamma(\beta+1)-\lambda \sigma^{\beta}-b \beta \xi^{\beta-1}} \times \\
& \cdot \int_{0}^{+\infty}\left[\psi_{1}(s)+\psi_{2}(s)\right]\left(1+s^{1-\beta}\right) \mathrm{d} s .
\end{aligned}
$$

Consequently,

$$
\begin{aligned}
\| v & -u \|_{E} \\
& \leq \frac{\Gamma(\beta+1)-\lambda \sigma^{\beta}-b \beta \xi^{\beta-1}}{\Gamma(\beta+1)-\lambda \sigma^{\beta}-b \beta \xi^{\beta-1}-\beta \int_{0}^{+\infty}\left[\psi_{1}(s)+\psi_{2}(s)\right]\left(1+s^{\beta-1}\right) \mathrm{d} s} \varepsilon .
\end{aligned}
$$

Thus, we get the Ulam-Hyers stability of (5)-(6). Then, if we take $C_{f}(\varepsilon)$ equal to the right hand side of (52), we obtain the generalized Ulam-Hyers stability of (5)-(6).

Theorem 3. Assume that the hypotheses $\left(A_{1}\right)$ and $\left(A_{2}\right)$ hold. In addition, the following hypotheses hold:

$$
\begin{aligned}
& \psi_{1}(t) \leq \frac{p}{\|v-u\|_{E}} \Phi(t), \\
& \psi_{2}(t) \leq \frac{q}{\|v-u\|_{E}} \Phi(t) .
\end{aligned}
$$

$\left(A_{5}\right)$ There exists a positive real number $C_{\Phi}$ such that, for each $t \in[0,+\infty)$, we have

$$
\Phi(t) \leq \int_{0}^{\infty}\left(1+s^{\beta-1}\right) \Phi(s) \mathrm{d} s \leq C_{\Phi} \Phi(t) .
$$

Then, problems (5) and (6) are generalized Ulam-Hyers-Rassias stable.

Proof. By exploiting the assumptions $\left(A_{2}\right),\left(A_{3}\right),\left(A_{4}\right)$, and $\left(A_{5}\right)$, then we get

$$
\begin{aligned}
|v(t)-u(t)| \leq \mid & |v(t)-P v(t)|+|P v(t)-u(t)| \\
\leq & |\mathscr{F} v(t)|+|P v(t)-P u(t)| \\
\leq & |\mathscr{F} v(t)| \\
& +\left|\int_{0}^{+\infty} H(t, s)[f(s, v(s))-f(s, u(s))] \mathrm{d} s\right| \\
& +\left|\int_{0}^{+\infty} H(t, s) \theta(s)[g(v(s))-g(u(s))] \mathrm{d} s\right| .
\end{aligned}
$$

$\left(A_{4}\right)$ There exist two positive constants $p$ and $q$ such that

Then,

$$
\begin{aligned}
\|u-v\|_{E} \leq & \|\mathscr{F} v\|_{E}+\left|\int_{0}^{\infty} \frac{H(t, s)}{1+t^{1-\beta}}[f(s, v(s))-f(s, u(s))] \mathrm{d} s\right| \\
& +\left|\int_{0}^{\infty} \frac{H(t, s)}{1+t^{1-\beta}} \theta(s)[g(v(s))-g(u(s))] \mathrm{d} s\right| \\
\leq & \Phi(t)+\frac{\beta}{\Gamma(\beta+1)-\lambda \sigma^{\beta}-b \beta \xi^{\beta-1}} \int_{0}^{+\infty}|f(s, v(s))-f(s, u(s))| \mathrm{d} s \\
& +\frac{\beta}{\Gamma(\beta+1)-\lambda \sigma^{\beta}-b \beta \xi^{\beta-1}} \int_{0}^{+\infty} \theta(s)|g(v(s))-g(u(s))| \mathrm{d} s
\end{aligned}
$$




$$
\begin{aligned}
\leq & \int_{0}^{\infty}\left(1+s^{\beta-1}\right) \Phi(s) \mathrm{d} s+\frac{\beta}{\Gamma(\beta+1)-\lambda \sigma^{\beta}-b \beta \xi^{\beta-1}} \int_{0}^{+\infty}|f(s, v(s))-f(s, u(s))| \mathrm{d} s \\
& +\frac{\beta}{\Gamma(\beta+1)-\lambda \sigma^{\beta}-b \beta \xi^{\beta-1}} \int_{0}^{+\infty} \theta(s)|g(v(s))-g(u(s))| \mathrm{d} s \\
\leq & C_{\Phi} \Phi(t)+\frac{\beta(p+q)}{\Gamma(\beta+1)-\lambda \sigma^{\beta}-b \beta \xi^{\beta-1}} \int_{0}^{+\infty}(1+s)^{1-\beta} \Phi(s) \mathrm{d} s \\
\leq & \left(1+\frac{\beta(p+q)}{\Gamma(\beta+1)-\lambda \sigma^{\beta}-b \beta \xi^{\beta-1}}\right) C_{\Phi} \Phi(t) \\
= & C_{f, \Phi} \Phi(t) .
\end{aligned}
$$

Hence, problems (5) and (6) are generalized Example 1. Ulam-Hyers-Rassias stable.

$$
\begin{cases}D_{0^{+}}^{5 / 2} u(t)=\frac{e^{-t}+\sin (u(t))}{100\left(1+t^{2}\right)\left(1+t^{3 / 2}\right)}+\frac{1+\sin (u(t))}{100(1+t)^{2}\left(1+t^{3 / 2}\right)} & t \in[0,+\infty), \\ u(0)=0, u^{\prime}(0)=0, & D_{0^{+}}^{3 / 2} u(+\infty)=b u(1)+\lambda \int_{0}^{2} u(s) \mathrm{d} s .\end{cases}
$$

In this example, we have

$$
\begin{aligned}
f(t, u(t)) & =\frac{e^{-t}+\sin (u(t))}{100\left(1+t^{2}\right)\left(1+{ }^{t}\right)}, \\
g(u(t)) & =1+\sin (u(t)), \\
|f(t, u(t))-f(t, v(t))| & \leq \frac{1}{100\left(1+t^{2}\right)\left(1+t^{3 / 2}\right)}|u(t)-v(t)|, \\
|g(u(t))-g(v(t))| & \leq|u(t)-v(t)|, \\
L & =1, \\
\theta(t) & =\frac{1}{100(1+t)^{2}\left(1+t^{3 / 2}\right)}, \\
\psi_{1}(t) & =\frac{1}{100\left(1+t^{2}\right)\left(1+t^{3 / 2}\right)}, \\
\psi_{2}(t) & =\frac{1}{100(1+t)^{2}\left(1+t^{3 / 2}\right)} .
\end{aligned}
$$

Since $\xi$ and $\sigma$ are fixed, then $\lambda$ and $b$ are chosen so that hypothesis $\left(A_{1}\right)$ is satisfied. So, we have 


$$
\left\{\begin{array}{l}
b<\frac{\Gamma(\beta+1)-\lambda \sigma^{\beta}}{\beta \xi^{\beta-1}}, \\
\lambda<\frac{\Gamma(\beta+1)}{\sigma^{\beta}} .
\end{array}\right.
$$

In our example, we have $\beta=(5 / 2), \xi=1, \sigma=2$. Then,

$$
\left\{\begin{array}{l}
b<\frac{2(\Gamma(7 / 2)-4 \sqrt{2})}{5} \approx 0.19 \\
\lambda<\frac{\Gamma(7 / 2)}{4 \sqrt{2}} \approx 0.58
\end{array}\right.
$$

So, we can choose $\lambda=1 / 2$ and $b=1 / 10$.

By simple computation, we get

$$
\begin{aligned}
\beta \int_{0}^{+\infty}\left(1+t^{\beta-1}\right)\left[\psi_{1}(t)+\psi_{2}(t)\right] \mathrm{d} t & =\frac{5}{200} \int_{0}^{+\infty} \frac{\mathrm{d} t}{1+t^{2}}+\frac{5}{200} \int_{0}^{+\infty} \frac{\mathrm{d} t}{(1+t)^{2}} \\
& =\frac{5}{200}\left[\arctan (t)-\frac{1}{1+t}\right]_{0}^{+\infty} \\
& =\frac{5}{200}\left(\frac{\pi}{2}+1\right) \approx 0.06, \\
\Gamma(\beta+1)-\lambda \sigma^{\beta}-b \beta \xi^{\beta-1} & \approx 0.25 .
\end{aligned}
$$

Thus, $\left(A_{2}\right)$ is satisfied.

Now, it remains to verify $\left(A_{3}\right)$. We have

$$
\begin{aligned}
\int_{0}^{+\infty}\left[\phi_{1}(t)+\phi_{2}(t)\right] \mathrm{d} t & \leq \int_{0}^{+\infty}\left(\frac{1}{\left(1+t^{2}\right)}+\frac{1}{(1+t)^{2}}\right) \mathrm{d} t \\
& =\frac{\pi}{2}+1<+\infty
\end{aligned}
$$

All hypotheses of Theorem 1 are satisfied. Therefore, boundary value problems (5)-(6) has at least one solution in $\mathbb{R}$.

\section{Data Availability}

No data were used in this study.

\section{Conflicts of Interest}

The authors declare that they have no conflicts of interest.

\section{Authors' Contributions}

The authors contributed equally and significantly in writing this paper. All authors read and approved the final manuscript.

\section{References}

[1] B. Azzaoui, B. Tellab, and K. Zennir, "Positive solutions for integral nonlinear boundary value problem in fractional sobolev spaces," Mathematical Methods in the Applied Sciences, pp. 1-18, 2021.

[2] A. Boulfoul, B. Tellab, N. Abdellouahab, and K. Zennir, "Existence and uniqueness results for initial value problem of nonlinear fractional integro-differential equation on an unbounded domain in a weighted Banach space," Mathematical
Methods in the Applied Sciences, vol. 44, no. 5, pp. 3509-3520, 2021.

[3] R. Hilfer, Applications of Fractional Calculus in Physics, World Scientific Publishing Co., Inc., River Edge, NJ, USA, 2000.

[4] A. A. Kilbas, H. M. Srivastava, and J. J. Trujillo, Theory and Applications of Fractional Differential Equations, p. 539, Elsevier, Amsterdam, Netherlands, 2006.

[5] I. Podlubny, "Fractional differential equations," Mathematics in Science and Engineering, vol. 198, 1999.

[6] V. E. Tarasov, Fractional Dynamics, Nonlinear Physical Science, Springer, Heidelberg, Germany, 2010.

[7] X. Zhao and W. Ge, "Unbounded solutions for a fractional boundary value problems on the infinite interval," Acta Applicandae Mathematica, vol. 109, no. 2, pp. 495-505, 2010.

[8] G. Wang, A. Cabada, and L. Zhang, "An integral boundary value problem for nonlinear differential equation of fractional order on an unbounded domain," Journal of Integral Equations, vol. 26, no. 1, 2014.

[9] C. Shen, H. Zhou, and L. Yang, "On the existence of solutions to a boundary value problem of fractional differential equation on the infinite interval," Boundary Value Problems, vol. 2015, Article ID 241, 2015.

[10] D. H. Hyers, "On the stability of the linear functional equation," Proceedings of the National Academy of Sciences, vol. 27, no. 4, pp. 222-224, 1941.

[11] T. M. Rassias, "On the stability of the linear mapping in Banach spaces," Proceedings of the American Mathematical Society, vol. 72, no. 2, pp. 297-300, 1978.

[12] S. M. Ulam, "A collection of mathematical problems," in Interscience Tracts in Pure and Applied Mathematicsp. 150, Interscience Publishers, London, UK, 1960.

[13] D. H. Hyers, G. Isac, and T. M. Rassias, "Stability of functional equations in several variables," Progress in Nonlinear Differential Equations and Their Applications, vol. 34, p. 313, 1998.

[14] S.-M. Jung, Hyers-Ulam-Rassias Stability of Functional Equations in Mathematical Analysis, p. 256, Hadronic Press, Inc., Palm Harbor, FL, USA, 2001.

[15] M. R. Abdollahpour, R. Aghayari, and M. T. Rassias, "HyersUlam stability of associated Laguerre differential equations in 
a subclass of analytic functions," Journal of Mathematical Analysis and Applications, vol. 437, no. 1, pp. 605-612, 2016.

[16] S.-M. Jung, "Hyers-Ulam stability of linear differential equations of first order," Applied Mathematics Letters, vol. 17, no. 10, pp. 1135-1140, 2004.

[17] T. Miura, S. Miyajima, and S.-E. Takahasi, "A characterization of Hyers-Ulam stability of first order linear differential operators," Journal of Mathematical Analysis and Applications, vol. 286, no. 1, pp. 136-146, 2003.

[18] S. Abbas, M. Benchohra, J. Lagreg, A. Alsaedi, and Y. Zhou, "Existence and Ulam stability for fractional differential equations of Hilfer-Hadamard type," Advances in Difference Equations, vol. 2017, no. 1, Article ID 180, 2017.

[19] N. Abdellouahab, B. Tellab, and K. Zennir, "Existence and stability results of A nonlinear fractional integro-differential equation with integral boundary conditions," Kragujevac Journal of Mathematics, vol. 46, no. 5, pp. 685-699, 2022.

[20] A. Naimi, T. Brahim, and K. Zennir, "Existence and Stability results for the solution of Neutral fractional integro-differential equation with nonlocal conditions," Tamkang Journal of Mathematics, vol. 53, pp. 1-22, 2021.

[21] W. Wei, X. Li, and X. Li, "New stability results for fractional integral equation," Computers \& Mathematics with Applications, vol. 64, no. 10, pp. 3468-3476, 2012.

[22] X.-J. Yang, General Fractional Derivatives: Theory, Methods and Applicationsy, CRC Press, New York, NY, USA, 2019.

[23] X.-J. Yang, F. Gao, and Y. Ju, General Fractional Derivatives with Applications in Viscoelasticity, Academic Press, Cambridge, MA, USA, 2020.

[24] Y. Zhou, Basic Theory of Fractional Differential Equations, World Scientific, Singapore, Singapore, 2014.

[25] Y. Liu, "Existence and unboundedness of positive solutions for singular boundary value problems on half-line," Applied Mathematics and Computation, vol. 144, no. 2-3, pp. 543-556, 2003.

[26] S. Abbas, M. Benchohra, and G. M. N. Guerekata, Topics in Fractional Differential Equations, Springer, New York, NY, USA, 2012.

[27] I. A. Rus, "Ulam stability of ordinary differential equations," Studia Univ. "Bases,-Bolyai", Mathematica, vol. LIV, no. 4, pp. 125-133, 2009. 